\title{
Maternal Educational Level Determine Mother-to- child Transmission of HIV Among HIV-exposed Infants in Governmental Health Facility of Bahir Dar City, Northwest Ethiopia.
}

Minyichil Birhanu ( $\triangle$ minyichil.birhanu@bdu.edu.et)

Bahir Dar University

Temesgen Ergetie

Bahir Dar University

Tiwobista Tenna

Bahir Dar University

Tsehayinesh Ayana

Bahir Dar University

Workie Dessie

Bahir Dar University

Workensh Belay

Bahir Dar University

\section{Research Article}

Keywords: Mother-to-child transmission, prevention of mother-to-child transmission, antiretroviral therapy, HIV-exposed infants, DNA/PCR, HIV

Posted Date: January 4th, 2021

DOI: https://doi.org/10.21203/rs.3.rs-126079/v1

License: (c) (1) This work is licensed under a Creative Commons Attribution 4.0 International License. Read Full License 


\section{Abstract}

Background: Globally at the end of 2011,3.2 million children under the age of 15 were living with HIV, among these children $91 \%$ found in Africa. In Ethiopia, one of every three children born to these women is infected with HIV. This study was done to determine mother-to-child transmission of HIV infection and its factors among HIV-exposed infants on PMTCT service.

Method: An institutional-based cross-sectional study was conducted among randomly selected 423 HIV exposed infants on PMTCT service in Bahir Dar city public health facility. Data were collected through chart review by using a pre-tested and structured checklist. Data was entered into Epi-data version 3.1 and exported to SPSS version 20 for analysis. The dependent variable association with explanatory variables was determined using logistic regression. Statistical significance was considered at $p$-value $<0.05$ with $95 \% \mathrm{Cl}$.

Result: The proportion of mother-to-child transmission of HIV infection among HIV-exposed infants on PMTCT services was 9.9\%. Maternal educational status (AOR=3.196; 95\% Cl: 1.161-8.797), ANC follow-up (AOR=5.414; 95\% Cl: 1.860-15.761), age of infant at HIV infection confirmed (AOR=0.088; 95\% Cl: 0.0330.238 ), and maternal $C D 4$ count ( $A O R=3.162 ; 95 \% \mathrm{Cl}: 1.295-7.720)$ were factors significantly associated with mother-to-child transmission of HIV.

Conclusion: The overall proportion of mother-to-child transmission of HIV infection among HIV exposed infants on PMTCT service were significantly high. This was due to low maternal educational status, absence of ANC follow-up, age of the infant at HIV infection confirmed, and low maternal CD4 count. Therefore, promoting women's education, antenatal care, age of the infant at HIV test, and maintaining maternal CD 4 count should be a great concern for health policymakers and health service providers.

\section{Background}

Human immunodeficiency virus (HIV) is a virus that attacks the body's immune system. If HIV is not treated, it can lead to acquired immunodeficiency syndrome (AIDS) (1). HIV can be transmitted from mother to child vertically, sexually, through contaminated blood products, and intravenous drug abuse (2). Vertical transmission of HIV infection occurs during pregnancy, labor and delivery, and breastfeeding (3). In 2009, AIDS was a major global health problem, that has been estimated that globally 33.4 million people were living with $\operatorname{HIV}(4,5)$. At the end of 2011, 3.2 million under the age of 15 years children were living with HIV globally, among these children $91 \%$ found in Africa (6). Without intervention, $25-40 \%$ of HIV infection in children is acquired through vertical transmission (7). In the United States, perinatal transmission of HIV by the mother accounts for $80 \%$ of pediatric HIV infections. Sexual abuse of children and high-risk behaviors in adolescents also contribute to youth HIV infection (7).

The number of HIV infected children has increased radically in developing countries over the last 30 years since the first HIV cases were identified in 1981. This is due to that the number of HIV-infected women of childbearing age has climbed (8). However, great improvements have been made in the United States and 
other industrialized countries to control mother to child transmission of HIV. The World Health Organization (WHO) estimates that almost 2.5 million children were living with HIV infection as of 2009 (8). Newly HIV-infected children in 2009 alone were 370,000 (9), this is a fall of $24 \%$ from 5 years before (10). Globally, one million HIV-exposed infants are born with HIV-infected women every year (11). There are about 1.4 million HIV-positive pregnant women who contribute to more than 300,000 neonatal and fetal deaths each year globally (12).

The HIV infection rate in pregnant women is as high as $0.3 \%$. In untreated women with viral loads of less than 100 copies/ml, the perinatal HIV transmission rates are 25\% (13). Even though prophylactic interventions have decreased the vertical transmission of HIV, perinatal transmission continues to occur (14). This is generally due to missed opportunities for HIV prevention, especially among women who miss prenatal care or who are not being offered voluntary HIV counseling and testing during pregnancy. For the majority $40 \%$ of mothers of infants with perinatally acquired HIV infection, the infection was not detected before delivery (13).

The Center for Disease Control (CDC) estimates that in 2009, in the 40 states with confidential namebased HIV infection reporting, an estimated 131 infants acquired HIV infection through vertical transmission (15). In 2012, antiretroviral prophylaxis or treatment was over 900,000 HIV positive women, to get HIV/AIDS-free children (16). To achieve HIV/AIDS-free children WHO started to implement different strategies for the optimization of prevention of mother to child transmission (PMTCT) care and support. These strategies are options $A, B$, and $B^{+}(17)$.

Globally, the burden of HIV infection in children are high in 22 countries(18). Ethiopia is one of these highly burdened countries where one of every three children born from HIV-positive women. The government has taken measures to expand the prevention of mother-to-child transmission of HIV service by endorsing antenatal care free of charge (19). To eliminate HIV infection in children and keep mothers alive, a comprehensive package of interventions implemented. This package includes preventing women from HIV infection, protect unwanted pregnancy, PMTCT throughout pregnancy, provide skilled delivery, exclusive breastfeeding, and providing appropriate HIV treatment, care, and support for mother and infants (20).

Moreover, in 2013, all pregnant women have been considered eligible to start long-term antiretroviral therapy (ART). Through a package is called option $B+$ which has a great role to ensure prevention of at least 98 percent of mother to child transmission of HIV (21). According to the WHO 2010 report, the prevalence of HIV in infants who are born from HIV-positive mothers who attend both treatment and prophylaxis was $10.9 \%$ (4). Worldwide 3.2 million Children living with HIV are $91 \%$ live in sub-Saharan Africa; $6 \%$ living in Asia and Pacific; the remaining 3\% are situated in the rest of the world (16).

Infants are infected with HIV at least 1600 every day and more than 600,000 infants are infected by the virus annually mostly in developing countries mainly in sub-Saharan Africa (21). In Ethiopia an estimated 
$1.2 \%$ of pregnant women are living with HIV consequently one of every three children born to these women is being infected with HIV (21).

Interventions like the use of ART drugs infected pregnant women, safe delivery practices, and safe infant feeding, avoid unwanted pregnancy has helped to reduce mother to child transmission (MTCT) from 40$5 \%$ respectively (22).

The UNAIDS on the fast-track to an AIDS-free generation 2016 report showed that the rate of HIV positive infants was 2.9\% in Uganda, $4.1 \%$ in Namibia, and 3.3\% in Swaziland (23). In 2013, the database of UNAIDS showed that the prevalence of HIV among infants born to HIV-infected mothers was $2 \%$ in Botswana, 34\% in Congo, and 25\% in Ethiopia (24). A study conducted in Ethiopia in 2014 with exposed infants the result showed that MTCT prevalence was $4.16 \%$ (25). A different study has showen that socio-demographic factors, prenatal factors, intrapartum factors, postnatal factors, and PMTCT services affect MTCT (25-33).

Thusly, assessing the HIV status of infants and its contributing factors among infants who were delivered from HIV positive pregnant and lactating mothers and who were enrolled in PMTCT service, important for program planners, policymakers, governmental organization, and non-governmental organizations to design strategies and interventions to decrease MTCT of HIV. Therefore, this study aimed to determine the proportion of mother-to-child transmission of HIV among HIV-exposed infants on PMTCT services and its associated factors in public health facility of Bahir Dar city, Ethiopia, 2018.

\section{Methods}

\section{Study design and Period}

An institutional-based cross-sectional study was conducted from March to April 2018. Bahir Dar city is the capital of Amhara Regional State, which is $565 \mathrm{~km}$ far from the capital city of Ethiopia, Addis Ababa. The city has one referral hospital and six health centers that provide PMTCT services.

\section{Sample size determination and Sampling procedure}

The sample size of the study was determined by using a single population proportion formula:

$$
n=\frac{(z \alpha / 2)^{2} p(1-p)}{d^{2}}
$$

The proportion of mother-to-child transmission of HIV infection among HIV-exposed infants on PMTCT services (P) was taken as $50 \%$ and with the assumptions of a $95 \%$ confidence level and $5 \%$ margin of error. Based on this assumption and adding a $10 \%$ non-response rate, the final sample size was 423 .

Six health centers and one referral hospital that were providing PMTCT service were included in the study. The total sample size was proportionally allocated to all six health centers and one referral hospital 
based on the number of HIV-exposed infants who were enrolled at PMTCT service in the last six years from January 2012 to December 2017. Then a simple random sampling technique was employed based on medical registration numbers by using random computer generating numbers to select each HIVexposed infants on PMTCT service in each health institution.

\section{Data collection procedures and quality control}

The data were collected by five trained diploma nurses through medical chart review methods by using a standardized structured checklist from ART register book, ANC follow-up register book, PMTCT service registration logbook charts, and infant DBS tally sheets at health centers and hospitals. The checklist was pre-tested among 21 randomly selected medical charts at Bahir Dar health center. Based on the pretest minor modifications were made to the checklist, such as editing or replacement of words that were not easily understood by the data collectors. The principal investigators and two supervisors were responsible for monitoring the data collection process.

\section{Data Processing and Analysis}

The collected data were coded and entered into Epi data version 3.1 then exported to the Statistical Package for Social Science (SPSS) version 20 for analysis. The descriptive and analytical analysis was used to present results. Bivariate and multivariable logistic regression models were used to analyze factors related to mother-to-child transmission of HIV. Independent variables with a p-value of 0.2 and less in the bivariate analysis were entered into the multivariable analysis. The level of association between dependent and independent variables were interpreted by using adjusted odds ratios with $95 \%$ confidence intervals and a p-value of less than 0.05 were considered as statistically significant.

\section{Result}

\section{Socio-demographic characteristics of infants and their mothers}

A total of $423 \mathrm{HIV}$-exposed infants were involved in the study with a $100 \%$ response rate. The mean age of the mothers was $29.34 \pm 5.817$ years. The majority of the mothers $366(86.5 \%)$ were urban residents. Concerning the educational status of the mothers, the majority $238(56.3 \%)$ had secondary and above education. The majority of the mothers were 298 (70.4\%) married. Almost half of the infants 214 (50.6\%) were male (Table 1). 
Table 1

Socio-demographic characteristics of infants and mothers at public health facility in Bahir Dar, Ethiopia, 2018.

\begin{tabular}{|c|c|c|c|}
\hline Variables & & Frequency & Percent \\
\hline \multirow[t]{5}{*}{ Age of the mother in years } & $<21$ & 35 & 8.3 \\
\hline & $21-25$ & 84 & 19.9 \\
\hline & $26-30$ & 171 & 40.4 \\
\hline & $31-35$ & 56 & 13.2 \\
\hline & $>35$ & 77 & 18.2 \\
\hline \multirow[t]{2}{*}{ Residency of the mother } & Rural & 57 & 13.5 \\
\hline & Urban & 366 & 86.5 \\
\hline \multirow[t]{3}{*}{ Mothers level of education } & No formal education & 92 & 21.7 \\
\hline & Primary & 93 & 22.0 \\
\hline & Secondary and above & 238 & 56.3 \\
\hline \multirow[t]{4}{*}{ Occupation of the mother } & Housewife & 108 & 25.5 \\
\hline & Farmer & 30 & 7.1 \\
\hline & Day labourer & 96 & 22.7 \\
\hline & Civil servants & 189 & 44.7 \\
\hline \multirow[t]{3}{*}{ Marital status of the mother } & Married & 298 & 70.4 \\
\hline & Single & 80 & 19 \\
\hline & Divorced/widowed & 45 & 10.6 \\
\hline \multirow[t]{2}{*}{ Sex of the infant } & Male & 214 & 50.6 \\
\hline & Female & 209 & 49.4 \\
\hline
\end{tabular}

\section{Prenatal, Intrapartum, Postnatal characteristics of infants, mothers and their partner}

The majority of mothers of infants, $383(90.5 \%)$ had antenatal care follow-up. And most of the partners 354 (83.7\%) were tested for HIV. Among the total reviewed mothers the majority 396 (93.6\%) was delivered at the health facility. And from those who delivered at the health facility, half 199 (50.3\%) were delivered at the health center. Regarding the birth weight of infants, the majority $333(78.7 \%)$ of the infant had normal birth weight, 39 (9.2\%) and 29 (6.9\%) had low birth weight and high birth weight respectively. 
Three hundred ten (73.3\%) of infants were enrolled in ARV intervention at the age of $>6$ weeks. Four hundred one (94.8\%) of infants were feed breast and complimentary food at the first 6 months of life.

\section{ARV intervention and clinical factors}

Four hundred nineteen (99.1\%) of infants were on ARV intervention. The majority 408(96.5\%) of infants mother were on ARV intervention and majority of mother start ARV before labor and delivery 374 (91.6\%). Most of the mothers 293(69.3\%) were on TDF + 3TC + EFV regimen. The majority of infants 419(99.1\%) received ARV prophylaxis. The majority infants 346(82.6\%) had taken ARV prophylaxis for six weeks.

\section{Proportion of mother-to-child transmission among HIV exposed infants on PMTCT services}

Among the total HIV-exposed infants on PMTCT services, 42(9.9\%) of were HIV infected (Fig. 1).

\section{Factors associated with mother-to-child HIV transmission among HIV-exposed infants on PMTCT services}

The overall proportion of HIV-infected infant through mother to child transmission among HIV-exposed infants was $9.9 \%$ (95\% Cl: 7.1-13.0). The proportion of mother-to-child HIV transmission was statistically associated with maternal educational status, ANC follow-up, age of infants at serostatus confirmation, and maternal CD4 count. The infant of a mother with primary educational status was 3.196 times more infected by HIV than those infants of a mother who had educational status of secondary and above (AOR $=3.196 ; 95 \% \mathrm{Cl}: 1.161-8.797)$. The odds of HIV infected infant were 5.414 times higher in infants of mother who had no ANC follow-up as compared with the infant of mother with ANC follow-up (AOR = 5.414; 95\% Cl: 1.860-15.761). Infant whose age at HIV infection confirmed between 6-18 months were 91.2\% times less likely to be infected by HIV than those infants aged $>18$ months at HIV infection confirmed (AOR $=0.088 ; 95 \% \mathrm{Cl}: 0.033-0.238$ ). Infant of a mother with CD4 count $\leq 350 \mathrm{cell} / \mathrm{mm}^{3}$ were 3.162 times more likely infected by HIV than those infant born from mother with CD4 count > $350 \mathrm{cell} / \mathrm{mm}^{3}$ (AOR=3.162; 95\% Cl: 1.295-7.720) (Table 2). 
Table 2

Multivariable logistic regression analysis of factors associated with MTCT of HIV infection among HIV exposed infants on PMTCT service at public health facility in Bahir Dar city, Ethiopia, 2018.

HIV status of the $\quad 95 \% \mathrm{Cl}$ infant

Positive Negative
COR
P. value

\section{Residency}

Urban

32

334

$0.45(0.208-$

0.357(0.112-

0.082

Rural

10

47

1

1

\section{Level of education}

No formal education

12

80

2.081(0.9444.590)

AOR

Primary
Secondary and above
ANC follow-up
No
Yes
Age of the infant at HIV
infection confirmed

$<6$ month

7

12

1.604(0.512-

2.952(0.751-

0.121

2.459(1.148-

5.267)

3.196(1.161-

8.797)

$\begin{array}{ll}1.601(0.451- & 0.467\end{array}$

$5.687)$

1

6-18 month

$23 \quad 336$

4.307(1.964-

9.444)

5.414(1.860-

15.761)

0.002

31

352

1

1

\begin{tabular}{lcclll}
\hline 6-18 month & 23 & 336 & $\begin{array}{l}0.188(0.086- \\
0.412)\end{array}$ & $\begin{array}{l}0.088(0.033- \\
0.238)\end{array}$ & 0.0001 \\
> 18month & 12 & 33 & 1 & 1 & \\
Maternal CD4 Count & & & & & \\
$\leq 350$ & 22 & 126 & $\begin{array}{l}2.226(1.171- \\
4.231)\end{array}$ & $\begin{array}{l}3.162(1.295- \\
7.720)\end{array}$ & 0.011 \\
$>350$ & & & 1 & 1 & \\
\hline
\end{tabular}

Note: $\mathrm{COR}=$ Crude Odds Ratio, $\mathrm{AOR}=$ Adjusted Odds Ratio, $\mathrm{Cl}=$ Confidence Interval.

\section{Discussion}


This study showed that the overall proportion of mother-to-child HIV transmission among HIV exposed infants on PMTCT services was $9.9 \%$ (95\% Cl: 7.1-13.0). This finding is consistent with the studies conducted in Brazil 9.16\% (28); Amhara region, Ethiopia 10.1\% (34); and Gonder university referral hospital, Ethiopia 10\%(31). However, this result was higher than the UNAIDS report of 2016 in Uganda 2.9\%, Nambia 4.1\%, Swaziland 3.3\% (35), UNAIDS 2013 database in Botswana 2\%(24), China 4.8\%(26). This discrepancy might be due to attributed to the different options of PMTCT intervention(36). On the other hand, the result of this study was lower than the UNAIDS 2013 database of Congo 34\%(24), Ethiopia 25\% (24) and the study conducted in Dire Dewa 15.7\%(37), and Jimma 17\% (30). This might be due to the difference in methodology the current study is cross-sectional but the other studies are followup studies.

In this study, mother-to-child HIV transmission among HIV exposed infants on PMTCT services was significantly associated with maternal educational status, ANC follow-up, age of infants at serostatus confirmation, and maternal CD4 count. We found that maternal education was strongly associated with mother-to-child HIV transmission among HIV exposed infants on PMTCT services. Mother to child transmission of HIV is 3 times higher among infant of mothers with primary education as compared with those with secondary and above educational level. This was in agreement with studies $(38,39)$. This might be due to that as educational level increase the mother's knowledge about mother to child HIV transmission is high.

In our study, ANC follow-up was strongly associated with mother-to-child transmission of HIV, which was consistent with similar studies carried out in Ethiopia(40). The possible reason could be that one of the components of ANC service is health education and awareness creation about mother-to-child transmission of HIV. Age of the infant at HIV infection confirmation has strong association with mother to child transmission. This result indicated that the age for HIV confirmation is important specifically in developing countries most of HIV test is carried out by antibody test and this antibody test is used after 18 month. However, studies in Ethiopia $(39,41)$ showed no significant association with mother-to-child transmission of HIV.

Maternal CD4 count was identified as a factor contributing to mother-to-child transmission of HIV among study participants in this study. Infants from a mother with low CD 4 count $\leq 350 \mathrm{cells} / \mathrm{mm}^{3}$ ) were more likely to be infected by mother-to-child transmission of HIV. This was in agreement with studies conducted in Zimbabwe (42). This could be explained by the reason that those mothers with low CD4 count will have low immunity and high viral load which will increase the risk of mother-to-child HIV transmission. But there are other contradictory to reports from Ethiopia (41). On the other hand the studies in France (43), Brazil (44), and Malawi (45) showed no significant association with mother-tochild transmission of HIV.

Unlike other earlier studies (31,39-41), home delivery, mixed infant feeding, residency, marital status, and infants were without ARV prophylaxis did not show a statistically significant relationship with the 
outcome variable in the final model. This variation may be due to the difference in sample size, study area, study design, method of analysis or could be also attributed to the quality of data captured.

\section{Limitation of the study}

As we use secondary data, it was challenging to control inconsistencies and missing values. This study did not aim to differentiate in which period does MTCT occurred (prepartum, intrapartum, or postpartum). The fact that all potential factors were not included and assessed may affect generalization of predictors in this study. Despite these limitations to the best of our knowledge, this study presented primary results of the effectiveness of routine PMTCT interventions in public health institutions in the study area.

\section{Conclusion And Recommendation}

This study showed that high proportion of mother-to-child transmission HIV infection among exposed infants in the study area. Infections with HIV among HIV exposed infants were positively associated with maternal level of education, history of ANC follow up, Age of the infant at HIV infection confirmed, and maternal CD4 counts. The findings indicate the need to focus on strengthening prevention of mother to child transmission service in health institutions. Health institutions that provide PMTCT service better to focus on increasing maternal educational status, increasing ANC follow-up, early initiation of ART to recover CD 4 count, and increase availability of DNA-PCR test for children less than 18 months. Therefore, we recommend the health institutions and Health policy-makers still should give emphasis on strengthening of PMTCT services in all health sectors. We also recommend to researchers to conduct further longitudinal studies to explore factors associated with mother-to-child transmission of HIV among HIV exposed infants.

\section{Abbreviation And Acronyms}

3TC-Lamovudine

AIDS-Acquired Immune Deficiency Syndrome

ANC-Antenatal Care

ART-Antiretroviral treatment

ARV- Antiretroviral

CDC-Center for Disease Control

DBS-Dried blood spot

EDHS-Ethiopian Demographic and Health Survey

EFV-Efavirenze

Page 10/15 
EMDHS-Ethiopia mini Demographic and Health Survey

HIV-Human Immune Virus

MTCT-Mother to Child Transmission

PMTCT-Prevention of Mother to Child Transmission

SPSS-Statistical Package for Social Science

TDF-Tenofovir

WHO-World Health Organization

\section{Declarations}

\section{Ethics approval and consent to participate}

Ethical clearance was obtained from the Research Ethical Review Committee of Bahir Dar University College of Medicine and Health Sciences Department of Pediatrics and Child Health Nursing and all methods were performed per the relevant guidelines and regulations. Permission and supportive letters were obtained from the respective health center and hospital chief executive officer before data collection. Information was collected anonymously after obtaining written informed consent from each health center and hospital PMTCT focal persons by assuring confidentiality throughout the data collection period, along with that informed consent was obtained from all mothers whose data used.

\section{Consent for publication}

Not applicable.

\section{Availability of data and material}

The dataset used and/or analyzed in this study are available from the correspondent author on reasonable request.

\section{Competing interest}

We confirm that there is no competing of interest.

\section{Funding}

No funding body

\section{Authors' contribution}


Minyichil B., Tiwobista T., Tsehayinesh A., Workie D., and Workensh B. were involved in the design of the study, data analysis, and interpretation of the findings, report writing, and paper preparation. Temesgen E. was involved in the manuscript writing and reviewed the report. All authors read and approved the final paper.

\section{Acknowledgement}

We would like to offer our in-depth gratitude to the data collectors, health centers, and hospital workers, especially those who work in PMTCT clinics for their support.

\section{References}

1. CDC. HIV Basics: Centers for Disease Control and Prevention; July 14, 2020 [Available from: https://www.cdc.gov/hiv/basics/whatishiv.html.

2. Organization WH. HIV/AIDS. 6 July 2020.

3. Organization WH. Paediatric HIV and treatment of children living with HIV: World Health Organization.; 2018 [Available from: https://www.who.int/hiv/pub/paediatric/diagnosis-arvinfants/en/.

4. Organization WH, UNAIDS. AIDS epidemic update: December 2009: WHO Regional Office Europe; 2009.

5. Hogg RS, Weber AE, Craib KJ, Anis AH, O'shaughnessy MV, Schechter MT, et al. One world, one hope: the cost of providing antiretroviral therapy to all nations. Aids. 1998;12(16):2203-9.

6. Organization $\mathrm{WH}$. Consolidated guidelines on the use of antiretroviral drugs for treating and preventing HIV infection: recommendations for a public health approach: World Health Organization; 2016.

7. Organization. WH. Strategic Vision.: World Health Organization; 2011 [

8. Organization WH. Paediatric HIV and treatment of children living with HIV. Geneva: WHO. 2010.

9. UNAIDS. AIDS epidemic update, December 2009: World Health Organization; 2009.

10. Organization WH. Global report: UNAIDS report on the global AIDS epidemic 2010. Geneva: WHO. 2010.

11. Bailey RC, Kamenga MC, Nsuami MJ, Nieburg P, St Louis ME. Growth of children according to maternal and child HIV, immunological and disease characteristics: a prospective cohort study in Kinshasa, Democratic Republic of Congo. International journal of epidemiology. 1999;28(3):532-40.

12. Organization WH. March 2014 supplement to the 2013 consolidated guidelines on the use of antiretroviral drugs for treating and preventing HIV infection: recommendations for a public health approach. 2014.

13. Delia M Rivera REF. Pediatric HIV Infection: Medscape Drug Reference; Sep 13, 2017 [

14. Prevention CfDCa. Achievements in public health. Reduction in perinatal transmission of HIV infection-United States,1985-2005. MMWR Morb Mortal Wkly Rep. 2006;55(21):5. 
15. Prevention CfDCa. HIV/AIDS Surveillance Report 2004. US Department of Health and Human Services, Centers for Disease Control and Prevention. 2005.

16. Derebe G, Biadgilign S, Trivelli M, Hundessa G, Robi ZD, Gebre-Mariam M, et al. Determinant and outcome of early diagnosis of HIV infection among HIV-exposed infants in southwest Ethiopia. BMC research notes. 2014;7(1):309.

17. Ethiopia. Ethiopia Demographic and Health Survey. Addis Ababa, Ethiopia2016.

18. HIV/AIDS UNPo. Global Plan towards the Elimination of New HIV Infections among Children by 2015 and Keeping their Mothers Alive. Joint United Nations Programme on HIV/AIDS (UNAIDS); 2011.

19. Demographic E. Health survey 2011 central statistical agency Addis Ababa. Ethiopia ICF International Calverton, Maryland, USA. 2012.

20. EDHS M. Ethiopia Mini Demographic and Health Survey. Central Statistical Agency Addis Ababa, Ethiopia. 2014.

21. Organization WH. Consolidated guidelines on HIV prevention, diagnosis, treatment and care for key populations. Consolidated guidelines on HIV prevention, diagnosis, treatment and care for key populations. 2014.

22. Kagaayi J, Gray RH, Brahmbhatt H, Kigozi G, Nalugoda F, Wabwire-Mangen F, et al. Survival of infants born to HIV-positive mothers, by feeding modality, in Rakai, Uganda. PLoS One. 2008;3(12):e3877.

23. HIV/AIDS JUNPo. On the fast-track to an AIDS-free generation; the incredible journey of the global plan towards the elimination of new HIV infections among children by 2015 and keeping their mothers alive. Geneva: UNAIDS. 2016.

24. Hill A, Dauncey T, Levi J, Heath K, Pérez Casas C. Higher risks of mother-to-child HIV transmission in countries with lower HIV prevalence: UNAIDS 2013 results for 32 countries with generalised epidemics2015. 257-63 p.

25. Tadele T, Tamiso A, Tadele T. Incidences and predictors of HIV positivity among infants who born from HIV positive mother who have follow up at two hospitals of southern Ethiopia, 2014. Sci J Public Health. 2014;2(5):431-9.

26. Wang $Q$, Wang L, Fang L, Wang A, Jin X, Wang F, et al. Timely antiretroviral prophylaxis during pregnancy effectively reduces HIV mother-to-child transmission in eight counties in China: a prospective study during 2004-2011. Scientific Reports. 2016;6:34526.

27. Gouveia PAdC, da Silva GAP, de Albuquerque MdFPM. Predictors of loss to follow-up among children registered in an HIV prevention mother-to-child transmission cohort study in Pernambuco, Brazil. BMC Public Health. 2014;14:1232.

28. Mwendo EM, Mtuy TB, Renju J, Rutherford GW, Nondi J, Sichalwe AW, et al. Effectiveness of prevention of mother-to-child HIV transmission programmes in $\mathrm{K}$ ilimanjaro region, northern $\mathrm{T}$ anzania. Tropical medicine \& international health. 2014;19(3):267-74.

29. Wudineh F, Damtew B. Mother-to-Child Transmission of HIV Infection and Its Determinants among Exposed Infants on Care and Follow-Up in Dire Dawa City, Eastern Ethiopia2016. 1-6 p. 
30. Birlie B, Diriba T, Sisay K, Gurmessa A, Heb S, Tadesse M. Mother to Child HIV Transmission and Its Predictors among HIV-Exposed Infants: A Retrospective Follow-Up Study in Southwest Ethiopia2016.

31. Koye DN, Zeleke BM. Mother-to-child transmission of HIV and its predictors among HIV-exposed infants at a PMTCT clinic in northwest Ethiopia. BMC Public Health. 2013;13(1):398.

32. Berhan Z, Abebe F, Gedefaw M, Tesfa M. Prevalence of HIV and associated factors among infants born to HIV positive women in Amhara Region, Ethiopia. International journal of clinical medicine. 2014;5(08):464.

33. Olana T, Bacha T, Worku W, Tadesse BT. Early infant diagnosis of HIV infection using DNA-PCR at a referral center: an 8 years retrospective analysis. AIDS Research and Therapy. 2016;13(1):29.

34. Wudineh F, Damtew B. Mother-to-Child Transmission of HIV Infection and Its Determinants among Exposed Infants on Care and Follow-Up in Dire Dawa City, Eastern Ethiopia. AIDS Research and Treatment. 2016;2016.

35. UNAIDS P. On the Fast-Track to an AIDS-free generation. Geneva: UNAIDS. 2016.

36. AIDS GiaeoHa. PREVENTION OF MOTHER-TO-CHILD TRANSMISSION (PMTCT) OF HIV 2020 [updated 23 April 2020. Available from: https://www.avert.org/professionals/hivprogramming/prevention/prevention-mother-child.

37. Wudineh F, Damtew B. Mother-to-Child Transmission of HIV Infection and Its Determinants among Exposed Infants on Care and Follow-Up in Dire Dawa City, Eastern Ethiopia. AIDS Res Treat. 2016;2016:3262746.

38. Gourlay A, Birdthistle I, Mburu G, lorpenda K, Wringe A. Barriers and facilitating factors to the uptake of antiretroviral drugs for prevention of mother-to-child transmission of HIV in sub-Saharan Africa: a systematic review. Journal of the International AIDS Society. 2013;16(1):18588.

39. Berhan Z, Abebe F, Gedefaw M, Tesfa M, Assefa M, Tafere Y. Risk of HIV and associated factors among infants born to HIV positive women in Amhara region, Ethiopia: a facility based retrospective study. BMC Research Notes. 2014;7(1):876.

40. Ebuy H, Bekele A, Redae G. HIV testing, test results and factors influencing among infants born to HIV positive mothers in public hospitals of Mekelle City, North Ethiopia: a cross-sectional study. BMC Infectious Diseases. 2020;20(1):67.

41. Desta ML, Saravanan M, Hilekiros H, Kahsay AG, Mohamed NF, Gezahegn AA, et al. HIV prevalence and risk factors in infants born to HIV positive mothers, measured by dried blood spot real-time PCR assay in Tigray, Northern Ethiopia. BMC Pediatrics. 2019;19(1):257.

42. Ngwende S, Gombe NT, Midzi S, Tshimanga M, Shambira G, Chadambuka A. Factors associated with HIV infection among children born to mothers on the prevention of mother to child transmission programme at Chitungwiza Hospital, Zimbabwe, 2008. BMC Public Health. 2013;13(1):1181.

43. Tubiana R, Le Chenadec J, Rouzioux C, Mandelbrot L, Hamrene K, Dollfus C, et al. Factors associated with mother-to-child transmission of HIV-1 despite a maternal viral load $<500$ copies $/ \mathrm{ml}$ at delivery: a case-control study nested in the French perinatal cohort (EPF-ANRS C01). 2010;50(4):585-96. 
44. Martínez A, Hora VPd, Santos ALd, Mendoza-Sassi R, Von Groll A, Soares EA, et al. Determinants of HIV-1 mother-to-child transmission in Southern Brazil. 2006;78(1):113-21.

45. Kim MH, Ahmed S, Preidis GA, Abrams EJ, Hosseinipour MC, Giordano TP, et al. Low Rates of Motherto-Child HIV Transmission in a Routine Programmatic Setting in Lilongwe, Malawi. PloS one. 2013;8(5):e64979.

\section{Figures}

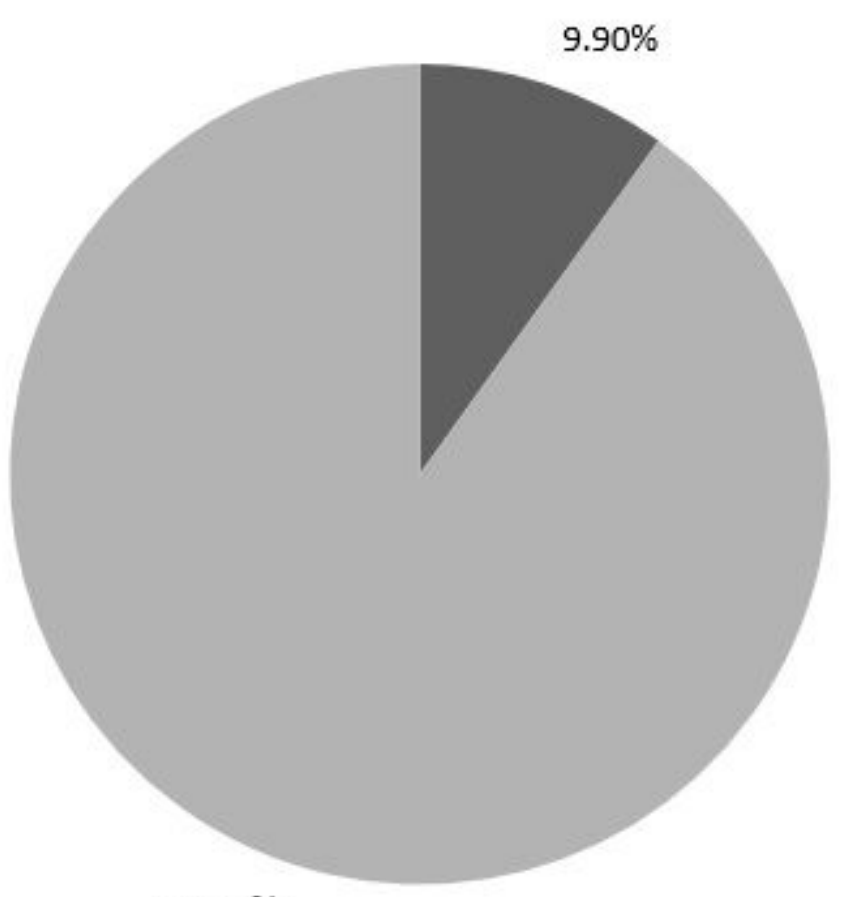

Positive

Negative

$90.10 \%$

\section{Figure 1}

HIV status of HIV-exposed infants on PMTCT services at public health facilities in Bahir Dar, Ethiopia, 2018. 